\title{
Discrimination of the rare medicinal plant Dendrobium officinale based on naringenin, bibenzyl, and polysaccharides
}

\author{
CHEN XiaoMei ${ }^{1 \dagger}$, WANG FangFei ${ }^{1 \dagger}$, WANG YunQiang ${ }^{2}$, LI XueLan ${ }^{2}$, WANG AiRong ${ }^{1}$, \\ WANG ChunLan ${ }^{1 *} \&$ GUO ShunXing ${ }^{1 *}$ \\ ${ }^{1}$ Institute of Medicinal Plant Development, Chinese Academy of Medical Sciences and Peking Union Medical College, Beijing 100193, China; \\ ${ }^{2}$ Institute of Medicinal Plant Development Yunnan Branch, Chinese Academy of Medical Sciences and Peking Union Medical College, \\ Jinghong 666100, China
}

Received July 6, 2012; accepted November 9, 2012

\begin{abstract}
The aim of this study was to establish a method for discriminating Dendrobium officinale from four of its close relatives Dendrobium chrysanthum, Dendrobium crystallinum, Dendrobium aphyllum and Dendrobium devonianum based on chemical composition analysis. We analyzed 62 samples of 24 Dendrobium species. High performance liquid chromatography analysis confirmed that the four low molecular weight compounds 4',5,7-trihydroxyflavanone (naringenin), 3,4-dihydroxy-4',5-dimethoxybibenzyl (DDB-2), 3',4-dihydroxy-3,5'-dimethoxybibenzyl (gigantol), and 4,4'-dihydroxy-3,3',5-trimethoxybibenzyl (moscatilin), were common in the genus. The phenol-sulfuric acid method was used to quantify polysaccharides, and the monosaccharide composition of the polysaccharides was determined by gas chromatography. Stepwise discriminant analysis was used to differentiate among the five closely related species based on the chemical composition analysis. This proved to be a simple and accurate approach for discriminating among these species. The results also showed that the polysaccharide content, the amounts of the four low molecular weight compounds, and the mannose to glucose ratio, were important factors for species discriminant. Therefore, we propose that a chemical analysis based on quantification of naringenin, bibenzyl, and polysaccharides is effective for identifying $D$. officinale.
\end{abstract}

Dendrobium officinale, naringenin, bibenzyl, polysaccharide, discriminant analysis

Citation: Chen X M, Wang F F, Wang Y Q, et al. Discrimination of the rare medicinal plant Dendrobium officinale based on naringenin, bibenzyl, and polysaccharides. Sci China Life Sci, 2012, 55: 1092-1099, doi: 10.1007/s11427-012-4419-3

There are 74 species and two varieties of Dendrobium in China [1], more than 50 of which can be used as 'Shihu', a popular tonic and a traditional Chinese medicine (TCM) [2]. Among them, Dendrobium officinale Kimura et Migo is the most endangered and precious species. It has been used for more than 2000 years in China as an antipyretic, nourishing Yin, benefiting the stomach, and promoting saliva production. In addition, D. officinale is widely recognized in South and Southeast Asia as a high-quality health food. Because of its pharmacodynamic effects, in the 2010 Edition of the Chinese Pharmacopoeia, D. officinale, divided from Den-

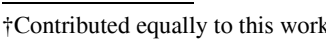

*Corresponding author (email: wangchunlan2006@163.com; sxguo2006@yahoo.com.cn) drobii Caulis ('Shihu'), is listed under the name Dendrobii Officinalis Caulis ('Tiepishihu') as the sole origin of the herb [3]. In China, D. officinale is now rare in the wild because of excessive collection and habitat damage. Almost all $D$. officinale materials are sourced from tissue culture or cultivated plants. Because of the limited supply, D. officinale products have a high market price, which encourages the proliferation of adulterated and substituted materials.

D. officinale shows strong morphological similarities to several of its close relatives; therefore, it is difficult to distinguish based on its appearance. Chemical analysis is highly sensitive and accurate for identifying the chemical components of natural materials; however, it has not been 
used to discriminate among Dendrobium species because of the lack of a well-accepted marker compound. All species in the genus contain polysaccharides, which are thought to be among the major active ingredients of $D$. officinale for enhancing immunity. The polysaccharides in some other medicinal plants in the genus also have immunomodulative effects [4-6]. In a previous study, D. officinale and its closely related species, such as Dendrobium aphyllum and Dendrobium crystallinum, could not be separated solely by their polysaccharide content and monosaccharide composition [7]. Besides polysaccharides, bibenzyl is relatively common in the genus and has neuro-protective and antitumor activities [8-10]. Phytochemical analyses of D. officinale have shown that it contains a wide variety of low molecular weight compounds including bibenzyls, phenanthrenes, and flavanones [11,12]. However, these have not been used to identify $D$. officinale, or for quality control of Dendrobii Officinalis Caulis.

The purpose of this work was to evaluate low molecular weight compounds and polysaccharides in Dendrobium species and determine whether there is a particular chemical composition signature that can be used to distinguish $D$. officinale from its four most common substitutes, D. aphyllum, D. crystallinum, Dendrobium chrysanthum, and Dendrobium devoniamum. We quantified eight compounds and polysaccharides and determined the monosaccharide composition of polysaccharides. Discriminant analysis was used for plant identification based on the chemical composition analyses. Clarifying the differences in chemical compositions among the Dendrobium species has practical and theoretical significance for making better use of $D$. officinale and its relatives.

\section{Materials and methods}

\subsection{Plant materials}

A total of 62 samples of 24 species were collected from three provinces of China, 61 of which were transplanted from their natural habitat or aseptic culture container into an artificial environment where they were cultivated for more than one year, and were collected from December 2008 to March 2009. Sample Offici-0 was collected from the Tianmu Mountains, Lin'an City, Zhejiang Province, in September 2006, and was used to validate the high performance liquid chromatography (HPLC) method. The details of collection sites are shown in Table 1. All samples were identified by Prof. Guo ShunXing. A sample of each batch was deposited at the Biotechnology Center, Institute of Medicinal Plant Development, Beijing.

Roots and leaves were removed, and the samples were cut into sections of $0.7-1.0 \mathrm{~cm}$. The samples were dried at $60^{\circ} \mathrm{C}$ for $3 \mathrm{~d}$ and then crushed and sieved through a 40 mesh sieve.

1.2 Instrumentation, operating conditions, and solution preparation for HPLC

The HPLC system (Waters 600, Milford, Massachusetts, USA) consisted of a quaternary gradient pump, column oven, and photodiode array detector (Waters 2996, Milford, Massachusetts, USA). Chromatographic data were acquired using Empower software. The experimental conditions were as follows: Kinetex $\mathrm{C}_{18}$ column $(100 \mathrm{~mm} \times 4.6 \mathrm{~mm}, 2.6 \mu \mathrm{m}$; Phenomenex, Los Angeles, California, USA), with gradient elution using (A) water with $5 \%$ acetonitrile and $0.1 \%$ trifluoroacetic acid and (B) acetonitrile as the mobile phase: 0 $\min (90 \% \mathrm{~A}) \rightarrow 10 \mathrm{~min}(80 \% \mathrm{~A}) \rightarrow 20 \mathrm{~min}(80 \% \mathrm{~A}) \rightarrow 30 \mathrm{~min}$

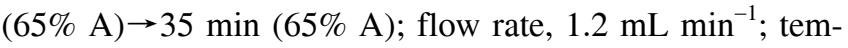
perature, $30^{\circ} \mathrm{C}$; detection wavelength, $230 \mathrm{~nm}$.

Eight marker compounds (provided by Dr. Yan Li) were as follows: (+)-syringaresinol-O- $\beta$-D-glucopyranoside (SG) [13], (2,3)-trans-N-( $p$-hydroxyphenethyl) ferulamide (moupinamide), 4',5,7-trihydroxyflavanone (naringenin) [14], and five bibenzyls: 4,4'-dihydroxy-3,3',5-trimethoxybibenzyl (moscatilin), 3',4-dihydroxy-3,5'-dimethoxybibenzyl (gigantol), 4,4'-dihydroxy-3,5-dimethoxybibenzyl (DDB-1), 3,4-dihydroxy-4',5-dimethoxybibenzyl (DDB-2) [15] and 3,4'-dihydroxy-5-methoxybibenzyl (DMB) [16] (Figure 1). Their purity, as assessed by HPLC, was $>98.0 \%$.

Standard solutions were prepared as follows: (i) for each compound, 1.00-2.00 mg was dissolved in $1.0 \mathrm{~mL}$ methanol as an individual stock solution; (ii) a mixed stock solution was prepared by combining $100 \mu \mathrm{L}$ of each stock solution and diluting the mixture to $1.0 \mathrm{~mL}$ with methanol. The precise concentration of SG, moupinamide, naringenin, moscatilin, gigantol, DDB-1, DDB-2, and DMB in the mixed stock solution was 128, 153, 107, 125, 175, 148, 173, and $177 \mu \mathrm{g} \mathrm{mL}^{-1}$, respectively.

Sample solutions were prepared as follows: (i) the sample was ultrasonically extracted with ethyl acetate for $1.5 \mathrm{~h}$; (ii) the extract solution was concentrated and then the residue was redissolved in methanol. For each sample, $20.0 \mu \mathrm{L}$ was loaded onto the Kinetex $\mathrm{C}_{18}$ column. Each sample solution was analyzed in triplicate.

\subsection{Calibration curves for HPLC}

For all compounds except for SG and DDB-2, the linearity was determined with six concentrations obtained by diluting the mixed stock solution 10-, 15-, 25-, 50-, 100- and 500 -fold with methanol. To determine the linearity of SG, the mixed stock solution was diluted 400-, 500-, 750-, 1000-, 2000- and 4000-fold, and for DDB-2, the individual stock solution was diluted 2.5-, 5-, 10-, 20-, 40-, and 200-fold. The injection volume was $10.0 \mu \mathrm{L}$. 
Table 1 Collection sites of 24 Dendrobium species

\begin{tabular}{|c|c|c|c|}
\hline Species & City/County & Province & Sample No. \\
\hline \multirow[t]{6}{*}{ D. officinale Kimura et Migo } & Mengyang & Yunnan & Offici-1, 2 \\
\hline & Menghai & Yunnan & Offici-3 \\
\hline & Pu'er & Yunnan & Offici- 4,5 \\
\hline & Zunyi & Guizhou & Offici- 6,7 \\
\hline & Pingbian & Yunnan & Offici-8, 9 \\
\hline & Jinhua & Zhejiang & Offici-10, 11 \\
\hline \multirow[t]{4}{*}{ D. devonianum Paxt. } & Jinghong & Yunnan & Devoni- $1^{\text {a) }}, 3,4$ \\
\hline & Menghai & Yunnan & Devoni-2 \\
\hline & Pingbian & Yunnan & Devoni-5 \\
\hline & Longling & Yunnan & Devoni-6, 7 \\
\hline D. chrysanthum Lindl. & Jinghong & Yunnan & Chrysa- $1,2^{\text {a) }}, 3,4$ \\
\hline \multirow[t]{2}{*}{ D. crystallinum Rchb. f. } & Jinghong & Yunnan & Crysta- 1,2 \\
\hline & Menghai & Yunnan & Crysta- $3^{\text {a) }}, 4^{\text {a) }}$ \\
\hline D. aphyllum (Roxb.) C.E. & Mengla & Yunnan & Aphyll-2, 3, 4 \\
\hline D. nobile Lindl. & Jinghong & Yunnan & Nobile- $1,2^{\text {a) }}, 3^{\text {a) }}$ \\
\hline D. chrysotoxum Lindl. & Jinghong & Yunnan & Chryso- $1^{\text {a) }}, 2^{\text {a) }}$ \\
\hline D. thyrsiflorum Rchb. f. & Jinghong & Yunnan & Thyrsi- $1,2^{\text {a) }}$ \\
\hline D. williamsonii Day et Rchb. f. & Jinghong & Yunnan & Willia- $1,2^{\text {a) }}$ \\
\hline D. longicornu Lindl. & Longling & Yunnan & Longic- $1,2^{\text {a) }}$ \\
\hline D. wardianum Warner & Jinghong & Yunnan & Wardia- $1,2^{a)}$ \\
\hline D. aurantiacum var. denneanum (Kerr) Z.H. Tsi & Jinghong & Yunnan & Aurant-1, $2^{\text {a) }}$ \\
\hline D. heterocarpum Lindl. & Menglian & Yunnan & Hetero- $1,2^{\text {a) }}$ \\
\hline D. crepidatum Lindl. ex Paxt. & Jinghong & Yunnan & Crepid-1, $2^{\text {a) }}$ \\
\hline D. moniliforme (L.) Sw. & Xinping & Yunnan & Monili-1, 2a) \\
\hline D. brymerianum Rchb. f. & Menghai & Yunnan & Brymer-1, $2^{\text {a) }}$ \\
\hline D. stuposum Lindl. & Jinghong & Yunnan & Stupos ${ }^{\text {a) }}$ \\
\hline D. acinaciforme Roxb. & Jinghong & Yunnan & $\operatorname{Acinac}^{a)}$ \\
\hline D. hercoglossum Rchb. F & Jinghong & Yunnan & $\operatorname{Hercog}^{\text {a) }}$ \\
\hline D. gratiosissimum Rchb. f. & Jinggu & Yunnan & Gratio $^{\text {a) }}$ \\
\hline D. falconeri Hook. & Longling & Yunnan & Falcon ${ }^{\text {a) }}$ \\
\hline D. guangxiense S.J. Cheng et C.Z. Tang & Malipo & Yunnan & Guangx $^{\text {a) }}$ \\
\hline D. fimbriatum Hook. & Malipo & Yunnan & Fimbri $^{\text {a) }}$ \\
\hline
\end{tabular}

a) Wild plant cultivated in artificial environment for more than one year.<smiles>COc1cc(CCc2ccc(OCCO)c(OC)c2)ccc1O</smiles>

moupinamide

naringenin

moscatilin<smiles>COc1cc(O)cc(CCc2ccc(O)c(OC)c2)c1</smiles>

gigantol

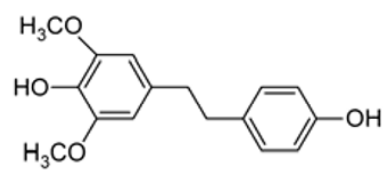

4,4'-dihydroxy-3,5dimethoxybibenzyl<smiles>COc1ccc(CCc2cc(O)c(O)c(OC)c2)cc1</smiles>

3,4-dihydroxy-4',5dimethoxybibenzyl

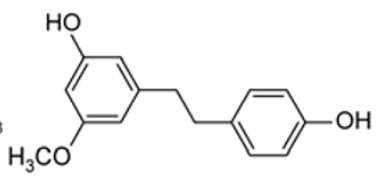

3,4'-dihydroxy-5 methoxybibenzyl

Figure 1 Structures of eight marker compounds. 


\subsection{HPLC method validation}

The limit of detection (LOD) and the limit of quantification (LOQ) were determined on the basis of a signal to noise ratio $(\mathrm{S} / \mathrm{N})$ of $3: 1$ and $10: 1$, respectively. The individual stock solution of each corresponding standard was sequentially diluted. The injection volume was $20.0 \mu \mathrm{L}$.

Intra-day precision was determined by calculating the relative standard deviation (RSD) of the peak area and the retention time of the diluted mixed stock solution among six consecutive HPLC runs each day. In inter-day precision was determined on six consecutive days. The variation was calculated using the RSD of six injections. The injection volume was $20.0 \mu \mathrm{L}$.

The precision of the method was evaluated by carrying out six independent assays of the Offici-0 solution. The accuracy of the method was evaluated by adding a known amount of each standard solution to the sample solution of Offici-0 (six replicates). The spiked and non-spiked samples were extracted and analyzed. The recovery was estimated as follows:

$$
\begin{aligned}
& \text { Recovery }(\%)=\left(C_{\mathrm{s}}-C_{\mathrm{ns}}\right) / C_{\mathrm{a}} \times 100 \\
& C_{\mathrm{s}}: \text { calculated concentration in spiked sample } \\
& C_{\mathrm{ns}}: \text { calculated concentration in non-spiked sample } \\
& C_{\mathrm{a}}: \text { concentration added }
\end{aligned}
$$

\subsection{Extraction and determination of polysaccharides}

The powdered materials were extracted in sequence with petroleum ether, methanol, and distilled water. An aliquot of the water extract was precipitated with four volumes of ethanol, kept at $4^{\circ} \mathrm{C}$ for $24 \mathrm{~h}$, and then centrifuged at $5180 \times g$ at $4^{\circ} \mathrm{C}$ for $15 \mathrm{~min}$. The precipitate was dissolved in distilled water for determination of polysaccharides using the phenol-sulfuric acid method [17]. Extractions and analyses were carried out in triplicate.

The rest of the water extract was condensed and then the subjected to the extraction procedure described above. The final precipitate was dissolved in distilled water, deproteinized with Sevag reagent [18], and then dialyzed (MWCO 7000) against deionized water for $48 \mathrm{~h}$. The solution inside the dialysis tubing was freeze-dried to yield the polysaccharide fraction for monosaccharide composition analysis.

\subsection{Analysis of monosaccharide composition}

Alditol acetate gas chromatography (GC) was used to analyze the monosaccharide composition of polysaccharides [19]. Monosaccharide standards (rhamnose, arabinose, xylose, mannose, galactose, and glucose; $>99.0 \%$ purity; purchased from the National Institute for Food and Drug Control, China) and polysaccharide hydrolysate were treated consecutively by deoxidation and acetylization. The final product was dissolved in chloroform.

For GC analysis, 1.0 $\mu \mathrm{L}$ supernatant was loaded onto an
Agilent DB-1701 capillary column (30 m×0.25 mm $\times 0.25$ $\mu \mathrm{m}$, Palo Alto, California, USA) of an Agilent $6890 \mathrm{~N}$ GC (Dual FID, Palo Alto, California, USA). The GC conditions

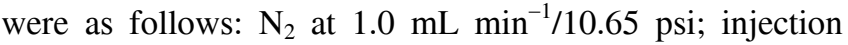
temperature, $230^{\circ} \mathrm{C}$; detector temperature, $250^{\circ} \mathrm{C}$; column temperature program: $180^{\circ} \mathrm{C}$ for $1 \mathrm{~min}$, increased to $250^{\circ} \mathrm{C}$ at $10^{\circ} \mathrm{C} \mathrm{m^{-1 }}$ and held for $5 \mathrm{~min}$, and then increased to $270^{\circ} \mathrm{C}$ at $10^{\circ} \mathrm{C} \mathrm{m^{-1 }}$ and held for $10 \mathrm{~min}$. Relative quantitative values (percentage monosaccharide composition) were obtained from the total peak area of six standards. Values shown in tables are the average of three repetitions.

\subsection{Statistical analysis}

SPSS 13.0 for Windows (SPSS Inc., 2005) was used for statistical analysis. Missing values were replaced with the mean value of a corresponding group. All data were log-transformed. Stepwise discriminant analysis (SDA) was applied to classify plants. Leave-one-out cross-validation was used to assess the predictive reliability of the discriminant analysis.

\section{Results}

\subsection{Calibration and method validation of HPLC}

The calibration curves for eight marker compounds were expressed as $Y=a x+b$, where $x$ is the concentration, $Y$ is the peak area of the standards, and $a$ and $b$ are constants. The calibration curves, correlation coefficients $\left(r^{2}\right)$, the linearity range for standards, and the LOD and LOQ values are shown in Table 2. The results for intra- and inter-day precision for eight standards are shown in Table 3. Accuracy and precision results are shown in Table 4. Figure 2 shows HPLC chromatograms of the eight marker compounds and sample Offici-0. The results confirmed that the method was highly accurate and precise.

\subsection{HPLC analysis of eight marker compounds in Dendrobium}

HPLC analysis showed that naringenin was widely distributed among Dendrobium spp. (20 of 24 species) with the highest detection rate of $90.3 \%$ (56 of 62 samples) (Table S1). Bibenzyls were present in all of the tested plants except for $D$. williamsonii. Of the five bibenzyl compounds, DDB-2 was common in the Dendrobium genus (13 of 24 species) and was the only compound that was always present in D. officinale. Moscatilin and gigantol were the two most common compounds, being detected in 21 and 19 of 24 tested species, respectively. These three bibenzyl compounds accounted for more than $90 \%$ of the total bibenzyl compounds in 19 species including $D$. officinale, D. chrysanthum, and D. aphyllum. Therefore, the chemical compo- 
Table 2 Calibration data for eight marker compounds

\begin{tabular}{|c|c|c|c|c|c|}
\hline Compound & Calibration curve & $r^{2}$ & Linearity range $\left(\mu \mathrm{g} \mathrm{mL}^{-1}\right)$ & $\operatorname{LOD}^{\mathrm{a})}\left(\mu \mathrm{g} \mathrm{mL}^{-1}\right)$ & $\mathrm{LOQ}^{\mathrm{b})}\left(\mu \mathrm{g} \mathrm{mL}^{-1}\right)$ \\
\hline $\mathrm{SG}^{\mathrm{c})}$ & $Y=20353 x-2128$ & 0.9993 & $0.32-3.20$ & 0.340 & 0.912 \\
\hline Moupinamide & $Y=18671 x+7357$ & 0.9991 & $0.30-15.30$ & 0.028 & 0.226 \\
\hline Naringenin & $Y=45689 x+8792$ & 0.9995 & $0.24-10.70$ & 0.026 & 0.214 \\
\hline Moscatilin & $Y=22076 x-651$ & 0.9998 & $0.15-12.50$ & 0.250 & 0.750 \\
\hline Gigantol & $Y=24822 x+7575$ & 0.9994 & $0.35-17.50$ & 0.087 & 0.350 \\
\hline DDB- $1^{\mathrm{d})}$ & $Y=36705 x-600$ & 0.9999 & $0.29-14.80$ & 0.148 & 0.592 \\
\hline $\mathrm{DMB}^{\mathrm{f})}$ & $Y=32435 x+14489$ & 0.9995 & $0.35-17.70$ & 0.088 & 0.354 \\
\hline
\end{tabular}

a) Limit of detection; b) limit of quantification; c) (+)-syringaresinol-O- $\beta$-D-glucopyranoside; d) 4,4'-dihydroxy-3,5-dimethoxybibenzyl; e) 3,4-dihydroxy-4',5-dimethoxybibenzyl; f) 3,4'-dihydroxy-5-methoxybibenzyl.

Table 3 Intra- and inter-day precision for quantification of eight marker compounds

\begin{tabular}{|c|c|c|c|c|c|c|c|c|}
\hline \multirow{2}{*}{ Compound } & \multicolumn{4}{|c|}{ Intra-day precision $(n=6)$} & \multicolumn{4}{|c|}{ Inter-day precision $(n=6)$} \\
\hline & Average area & $\operatorname{RSD}^{\mathrm{a})}(\%)$ & Average $\mathrm{Rt}^{\mathrm{b})}(\mathrm{min})$ & $\operatorname{RSD}(\%)$ & Average area & $\operatorname{RSD}(\%)$ & Average Rt (min) & RSD (\%) \\
\hline $\mathrm{SG}^{\mathrm{c})}$ & 2624608 & 0.76 & 8.06 & 1.36 & 2607365 & 1.42 & 8.01 & 1.43 \\
\hline Moupinamide & 5031508 & 0.42 & 12.71 & 0.92 & 4991796 & 1.97 & 12.67 & 1.01 \\
\hline Naringenin & 8460454 & 0.46 & 17.76 & 0.97 & 8410057 & 1.65 & 17.71 & 1.04 \\
\hline Moscatilin & 5068251 & 0.33 & 22.97 & 1.34 & 5041484 & 1.55 & 22.90 & 1.41 \\
\hline Gigantol & 7520248 & 0.68 & 25.56 & 1.01 & 7483326 & 1.58 & 25.50 & 1.06 \\
\hline DDB- $1^{\text {d) }}$ & 6673261 & 0.65 & 21.93 & 1.22 & 6641031 & 1.48 & 21.85 & 1.23 \\
\hline DDB- $2^{\mathrm{e})}$ & 4365939 & 2.55 & 30.68 & 0.46 & 4317410 & 2.31 & 30.65 & 0.48 \\
\hline $\mathrm{DMB}^{\mathrm{f})}$ & 8398002 & 0.92 & 24.20 & 1.20 & 8356092 & 1.68 & 24.13 & 1.26 \\
\hline
\end{tabular}

a) Relative standard deviation; b) retention time; c) (+)-syringaresinol-O- $\beta$-D-glucopyranoside; d) 4,4'-dihydroxy-3,5-dimethoxybibenzyl; e) 3,4-dihydroxy-4',5-dimethoxybibenzyl; f) 3,4'-dihydroxy-5-methoxybibenzyl.

Table 4 Accuracy and precision for quantification of eight marker compounds in sample Offici-0

\begin{tabular}{|c|c|c|c|c|}
\hline \multirow{2}{*}{ Compound } & \multicolumn{2}{|c|}{ Method accuracy $(n=6)$} & \multicolumn{2}{|c|}{ Method precision $(n=6)$} \\
\hline & Recovery (\%) & $\operatorname{RSD}^{\mathrm{a})}(\%)$ & Concentration $\left(\mu \mathrm{g} \mathrm{g}^{-1}\right)$ & $\operatorname{RSD}(\%)$ \\
\hline $\mathrm{SG}^{\mathrm{b})}$ & 95.60 & 1.52 & 2.09 & 0.76 \\
\hline Moupinamide & 101.50 & 1.63 & 13.29 & 1.33 \\
\hline Naringenin & 99.50 & 1.41 & 27.33 & 1.69 \\
\hline Moscatilin & 99.00 & 1.30 & 33.21 & 2.50 \\
\hline Gigantol & 95.50 & 1.87 & 21.60 & 1.14 \\
\hline DDB- $2^{\mathrm{d})}$ & 96.00 & 1.95 & 146.62 & 0.81 \\
\hline $\mathrm{DMB}^{\mathrm{e})}$ & 98.60 & 2.02 & 33.33 & 2.60 \\
\hline
\end{tabular}

a) Relative standard deviation; b) (+)-syringaresinol-O- $\beta$-D-glucopyranoside; c) 4,4'-dihydroxy-3,5-dimethoxybibenzyl; d) 3,4-dihydroxy-4',5dimethoxybibenzyl; e) 3,4'-dihydroxy-5-methoxybibenzyl.

sition data were useful to separate $D$. officinale from its four close relatives.

In the cultivated $D$. officinale samples, moscatilin and gigantol were found only rarely (in 2 out of 11 samples; Offici-4 and Offici-5 from Pu'er, Yunnan Province). Therefore, it is unclear whether moscatilin and/or gigantol are characteristic compounds of this species. Furthermore, Offici-4 and Offici-5 differed greatly from other samples in their diversity of bibenzyl compounds. Their bibenzyl composition appeared to be specific to the production area. A similar trend was also observed in $D$. devonianum. The samples from Pingbian and Longling contained only gigantol and DDB-2; while those from Jinghong and Menghai contained 4-5 bibenzyl compounds. This result was consistent with the assumption that besides genetic predisposition, the ecological environment plays an important role in the biosynthesis and accumulation of secondary metabolite in plants, thereby affecting or even determining the quality of TCM.

There were 16 species for which we had two or more samples; for most of them, the content of a particular bibenzyl was higher in wild samples than in cultivated ones. These results suggested that an extended growth period promotes accumulation of bibenzyls in Dendrobium. Further research should be conducted to clarify the relationship between bibenzyls and the efficacy of medicinal Den- 

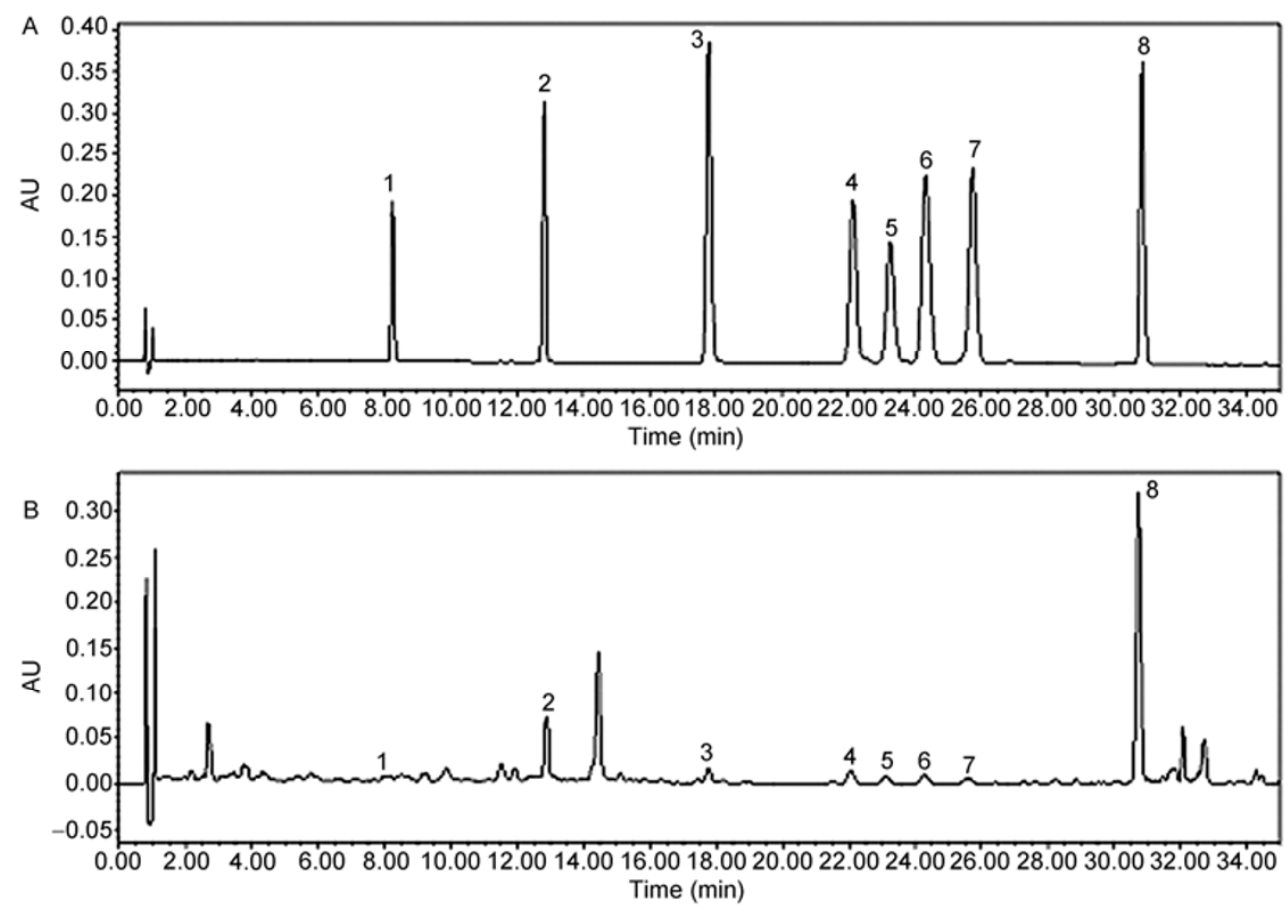

Figure 2 HPLC chromatograms. A, Chromatogram of the eight marker compounds: (+)-syringaresinol-O- $\beta$-D-glucopyranoside (SG) (peak 1), moupinamide (peak 2), naringenin (peak 3), 4,4'-dihydroxy-3,5-dimethoxybibenzyl (DDB-1) (peak 4), moscatilin (peak 5), 3,4'-dihydroxy-5-methoxybibenzyl (DMB) (peak 6), gigantol (peak 7), and 3,4-dihydroxy-5,4'-dimethoxybibenzyl (DDB-2) (peak 8). B, Chromatogram of sample Offici-0.

drobium plants. Such information would be useful for selection and for quality control.

\subsection{Polysaccharide analysis}

The polysaccharide content and monosaccharide composition of polysaccharides are shown in Table S2. Mannose or glucose was the most abundant monosaccharide in the polysaccharide in many of the Dendrobium species. These monosaccharides were ubiquitous in all samples and made up more than $90 \%$ of the total polysaccharides in 22 species (all tested species except $D$. fimbriatum and $D$. acinaciforme). In the 31 samples of $D$. officinale (including Offici-0), D. aphyllum, D. crystallinum, D. chrysanthum, and $D$. devoniamum, the relative contents of mannose and glucose were more consistent than total polysaccharide content $(\mathrm{PC})$. This result indicated that the mannose to glucose ratio $\left(R_{\mathrm{M} / \mathrm{G}}\right)$ might be an important characteristic of each plant. Therefore, in addition to $\mathrm{PC}, R_{\mathrm{M} / \mathrm{G}}$ was used as a variable in the discriminant analysis.

There was no particular pattern in total PC in D. officinale and $D$. devonianum as related to their production area. Because most of the samples were tissue-cultured seedlings from different batches and/or companies, the differences in total PC may have been due to different seedling germplasms. Furthermore, unlike naringenin and bibenzyl, the levels of monosaccharides and polysaccharides were not related to the growth period.

\subsection{Discriminant analysis}

A stepwise discriminant analysis was applied to select which variables best differentiated among the five closely related Dendrobium species; no variable was removed from the analysis. The partial Wilks' lambda test indicated that $R_{\mathrm{M} / \mathrm{G}}(F=137.4)$ and DDB-2 $(F=112.9)$ were the two most significant variables with the greatest discriminatory power, followed by naringenin $(F=98.5)$, giganol $(F=97.4)$, moscatilin $(F=78.9)$, and PC $(F=66.8)$. To determine which species a sample belonged to, five classification functions were created, allowing $100 \%$ correct classification of all samples. The scatter plot between the first two discriminant functions grouped samples according to species (Figure 3). Cross-validation upheld the classification results, which justified the discriminatory power of the technique. Using two types of components, our approach distinguished $D$. officinale from four of its closest relatives based on chemical analyses of naringenin, bibenzyl, and polysaccharides.

\section{Discussion}

The results of discriminant analysis demonstrated the discriminatory power of four low molecular weight compounds for identification of $D$. officinale. Naringenin is the predominant flavanone in grapefruit and has antioxidant, anti-inflammatory, and antitumor activities [20]. This com- 


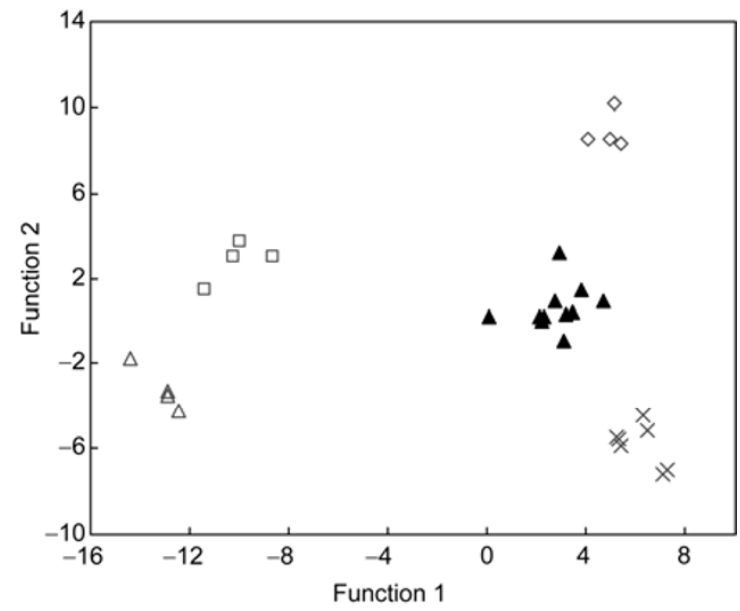

Figure 3 Discriminant analysis of $D$. officinale and four close relatives. Scatter plot of the first two discriminant functions discriminated among $D$. officinale (closed triangles), D. chrysanthum (open squares), D. crystallinum (open diamonds), D. aphyllum (open triangles) and D. devonianum (crosses).

pound has been isolated from $D$. dendiflorum and $D$. trigonopus [21,22], and in this study, was present in all tested Dendrobium species. Our results showing the wide distribution of moscatilin and giganol in Dendrobium as major bibenzyl constituents were similar to those of Yang and colleagues [8]. There are few reports about DDB-2; it is present in D. moniliforme and D. gratiosissimum [23,24] but little is known about its biological activity. Our research showed that DDB-2 is a common constituent of Dendrobium, especially D. officinale, D. devonianum, and D. chrysanthum.

There have been some reports on identification of $D$. officinale based on chemical composition [7,25-27]; however, no particular low molecular weight compound was identified as a marker in those studies. In fact, except for polysaccharides, the effective and/or characteristic constituents of $D$. officinale were uncertain. In this research, naringenin, moscatilin, gigantol, and DDB-2 were important for identification of $D$. officinale. Therefore, these four compounds are significant for quality control of Dendrobii Officinalis Caulis.

The monosaccharide profiles of polysaccharides have been used to identify fruit polysaccharides [28] and to discriminate among different traditional Chinese medicine and their origins $[7,25]$. The results of those studies indicated the monosaccharide profiles obtained after acid hydrolysis (i.e., amounts and composition of monosaccharides) were crucial for quality control of polysaccharides. Similarly, analysis of the monosaccharide profile has been used for quality control of Dendrobii Officinalis Caulis; the Chinese Pharmacopoeia (2010 Edition) notes that the ratio of mannose and glucose $\left(R_{\mathrm{M} / \mathrm{G}}\right)$ in Dendrobii Officinalis Caulis should be 2.4-8.0 [3]. In our research, the $R_{\mathrm{M} / \mathrm{G}}$ values of $D$. officinale consistent with that standard; however, those of $D$. crystallinum and D. aphyllum were also within the standard range, implying that the polysaccharide characters are inadequate to identify $D$. officinale. Therefore, we anticipated that a combination of polysaccharide and low molecular weight compositions would be more useful for identification. The results of the discriminant analysis demonstrated the discriminatory power of naringenin, bibenzyl, and polysaccharides for identifying $D$. officinale. These findings will be useful for further research on quality control of Dendrobii Officinalis Caulis or/and Dendrobii Caulis.

Owing to the huge market demands for both pharma foods and TCM made from $D$. officinale and its processed materials, these results have considerable practical implications for increasing the medicinal and health-promoting functions of $D$. officinale. As well, this approach will be useful for detecting and controlling falsification of Dendrobii Officinalis Caulis. These findings are preliminary, partly because relatively few samples were included in this study. In addition, the contents of some compounds varied widely, even among different samples of the same species. Further research should be conducted to evaluate the effects of various phenological factors, such as the production area and/or growth period, on chemical composition of Dendrobium.

This work was supported by the National Natural Science Foundation of China (Grant Nos. 30830117 and 31170016) and the Major Scientific and Technological Special Project for Significant New Drugs Creation (Grant No. 2012ZX09301002-001-031). We thank Dr. Liu ZhiHua from the Institute of Medicinal Plant Development for advice on statistical analysis. We also thank Ms. Li Ye, from Beijing Foreign Studies University, for advice on the manuscript.

1 Tsi Z H, Chen S C, Luo Y B, et al. Orchidaceae (3). In: Tsi Z H, ed. Angiospermae, Monocotyledoneae, Flora Reipublicae Popularis Sinica (in Chinese). Beijing: Science Press, 1999. 67-146

2 Bao X S, Shun Q S, Zhang S H, et al. Chinese Medicinal Dendrobium Atlas (in Chinese). Shanghai: Shanghai Scientific and Technological Literature Publishing House, 2005

3 Pharmacopoeia of China, Part I (in Chinese). 2010 ed. Beijing: China Medical Science Press, 2010. 265-266

4 Hsieh Y S Y, Chien C, Liao S K S, et al. Structure and bioactivity of the polysaccharides in medicinal plant Dendrobium huoshanense. Bioorgan Med Chem, 2008, 16: 6054-6068

5 Fan Y, He X, Zhou S, et al. Composition analysis and antioxidant activity of polysaccharide from Dendrobium denneanum. Int $\mathrm{J}$ Bio Macromol, 2009, 45: 169-173

6 Wang J H, Luo J P, Zha X Q, et al. Comparison of antitumor activities of different polysaccharide fractions from the stems of Dendrobium nobile Lindl. Carbohyd Polym, 2010, 79: 114-118

$7 \mathrm{Xu} \mathrm{J}$, Guan J, Chen X J, et al. Comparison of polysaccharides from different Dendrobium using saccharide mapping. J Pharmaceut Biomed, 2011, 55: 977-983

8 Yang L, Wang Z, Xu L. Simultaneous determination of phenols (bibenzyl, phenanthrene, and fluorenone) in Dendrobium species by high-performance liquid chromatography with diode array detection. J Chromatogr A, 2006, 1104: 230-237

9 Song J X, Shaw P C, Sze C W, et al. Chrysotoxine, a novel bibenzyl compound, inhibits 6-hydroxydopamine induced apoptosis in $\mathrm{SH}-\mathrm{S} Y 5 \mathrm{Y}$ cells via mitochondria protection and NF- $\mathrm{KB}$ modulation. Neurochem Int, 2010, 57: 676-689

10 Tsai A C, Pan S L, Liao C H, et al. Moscatilin, a bibenzyl derivative 
from the India orchid Dendrobrium loddigesii, suppresses tumor angiogenesis and growth in vitro and in vivo. Cancer Lett, 2010, 292: 163-170

11 Li Y, Wang C L, Wang Y J, et al. Three new bibenzyl derivatives from Dendrobium candidum. Chem Pharm Bull, 2009, 57: 218-219

12 Li Y, Wang C L, Wang Y J, et al. Four new bibenzyl derivatives from Dendrobium candidum. Chem Pharm Bull, 2009, 57: 997-999

13 Wang F F, Li Y, Dong H L, et al. A new compound from Dendrobium candidum (in Chinese). Chin Pharm J, 2010, 45: 898-892

14 Li Y, Wang C L, Wang F F, et al. Phenolic components and flavanones from Dendrobium candidum (in Chinese). Chin Pharm J, 2010, 45: 975-979

15 Li Y, Wang C L, Guo S X, et al. Two new compounds from Dendrobium candidum. Chem Pharm Bull, 2008, 56: 1477-1479

16 Li Y, Wang C L, Wang F F, et al. Chemical constituents of Dendrobium candidum (in Chinese). China J Chin Mater Med, 2010, 35 : 1715-1719

17 Dubois M, Gilles K A, Hamilton J K, et al. Colorimetric method for determination of sugar and related substances. Anal Chem, 1956, 28: 350-356

18 Navarini L, Gilli R, Gombac V, et al. Polysaccharides from hot water extracts of roasted Coffea arabica beans: isolation and characterization. Carbohyd Polym, 1999, 40: 71-81

19 Li T L, Zhu A, Yu H N, et al. Gas chromatography of glycoconjugate. In: Zhang W J, ed. Biochemical Research Technology on Glycoconjugate (in Chinese). 2nd ed. Hangzhou: Zhejiang University Press, 2006. 36-47
20 Ross J A, Kasum C M. Dietary flavonoids: bioavailability, metabolic effects, and safety. Annu Rev Nutr, 2002, 22: 19-34

$21 \mathrm{Hu} \mathrm{J}$ M, Chen J J, Yu H, et al. Two novel bibenzyls from Dendrobium trigonopus. J Asian Nat Prod Res, 2008, 10: 653-657

22 Fan C, Wang W, Wang Y, et al. Chemical constituents from Dendrobium densiflorum. Photochemistry, 2001, 57: 1255-1258

23 Bi Z M, Wang Z T, Xu L S. Chemical constituents of Dendrobium moniliforme. Acta Bot Sin, 2004, 46: 124-126

24 Wang M, Zhang C F, Wang Z T, et al. Studies on constituents of Dendrobium gratiosissimum (in Chinese). China J Chin Mater Med, 2007, 32: 701-703

25 Kuang H, Xia Y, Liang J, et al. Fast classification and compositional analysis of polysaccharides from TCMs by ultra-performance liquid chromatography coupled with multivariate analysis. Carbohyd Polym, 2011, 84: 1258-1266

26 Zha X Q, Luo J P, Wei P. Identification and classification of Dendrobium candidum species by fingerprint technology with capillary electrophoresis. S Afr J Bot, 2009, 75: 276-282

27 Wang L, Wang C, Pan Z, et al. Application of pyrolysis-gas chromatography and hierarchical cluster analysis to the discrimination of the Chinese traditional medicine Dendrobium candidum Wall. ex Lindl. J Anal Appl Pyrol, 2011, 90: 13-17

28 Arnous A, Meyer A. Quantitative prediction of cell wall polysaccharide composition in grape (Vitis vinifera $\mathrm{L}$.) and apple (Malus domestica) skins from acid hydrolysis monosaccharide profiles. J Agr Food Chem, 2009, 57: 3611-3619

Open Access This article is distributed under the terms of the Creative Commons Attribution License which permits any use, distribution, and reproduction in any medium, provided the original author(s) and source are credited.

\section{Supporting Information}

Table S1 Content of eight marker compounds $\left(\mu \mathrm{g} \mathrm{g}^{-1}\right)$ in 62 samples of 24 Dendrobium species

Table S2 Polysaccharide content (PC) and monosaccharide composition analysis of 62 samples of 24 Dendrobium species

The supporting information is available online at life.scichina.com and www.springerlink.com. The supporting materials are published as submitted, without typesetting or editing. The responsibility for scientific accuracy and content remains entirely with the authors. 